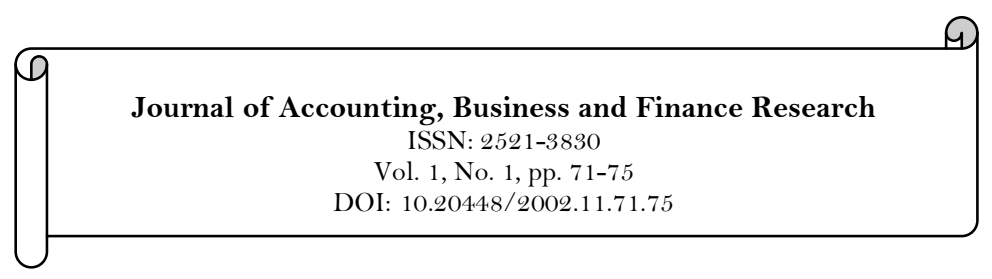

\title{
GARCH Model With Fat-Tailed Distributions and Bitcoin Exchange Rate Returns
}

\author{
Ruiping Liu ${ }^{1}$ \\ Zhichao Shao ${ }^{2}$ \\ Guodong Wei ${ }^{3}$ \\ Wei Wang ${ }^{4}$ \\ ${ }^{1}$ School of Economics, Guizhou University, Guiyang, Guizhou, China. \\ ${ }^{2}$ Business School, Guangxi University, Nanning, Guangxi, China. \\ ${ }^{3}$ Department of Economics, University of Utah, Salt Lake City, Utah, United States. \\ ${ }^{4}$ School of Economics, Guizhou University, Guiyang, Guizhou, China.
}

\begin{tabular}{|c|c|}
\hline Abstract & \\
\hline $\begin{array}{l}\text { In the era of diminishing power from US dollar and increasing } \\
\text { competition among world currencies, Bitcoin, as a completely new } \\
\text { concept as a medium of exchange, has received increasing attentions } \\
\text { over the world. Nowadays, Bitcoin also becomes an investment } \\
\text { vehicle, which carries attractive opportunities but also significant } \\
\text { risks for the investment community. In this paper, we have compared } \\
\text { the empirical performance of a newly-developed heavy-tailed } \\
\text { distribution, the normal reciprocal inverse Gaussian (NRIG), with } \\
\text { the most popular heavy-tailed distribution, the Student's t } \\
\text { distribution, under the GARCH framework in fitting the daily } \\
\text { Bitcoin exchange rate returns. Our results indicate the heavy-tailed } \\
\text { distribution has better performance in capture the daily Bitcoin } \\
\text { exchange rate returns dynamics than the standard normal } \\
\text { distribution. Our results also show the older fashioned Student's t } \\
\text { distribution still performs better than the new heavy-tailed } \\
\text { distribution. }\end{array}$ & $\begin{array}{l}\text { Licensed: } \\
\text { This work is licensed under a } \\
\text { Creative Commons Attribution } \\
\text { 4.0 License. } \\
\text { Publisher: } \\
\text { Scientific Publishing Institute }\end{array}$ \\
\hline
\end{tabular}

\section{Introduction}

Since the creation of Bitcoin in 2009, this cryptocurrency has become more and more popular over the world. People from developed countries to developing countries and from big cities to small village are attracted to invest this new investment vehicle. Using Bitcoin, people have their transactions taking place between each other directly, without an intermediary. The exchange rate of Bitcoin to USD is currently above $\$ 2700$, starting from almost nothing in 2010. The market capitalization has been over $\$ 46$ billions and is considered to continue growing. The number of active Bitcoin users has increased significantly since its introduction to the market.

There are a lot of economists who are quite interested in the economics of Bitcoin. Although Bitcoin was invented as a medium of exchange, it is now more as an investment tool since many speculative investors buy Bitcoin for its investment value rather than as a currency. Because Bitcoin lacks of guaranteed value and digital nature, it means that the purchase and use of Bitcoin has significant financial risks. Moreover, the regulators do not have efficient tools to regulate the market as a lack of sufficient experience. This means investment of Bitcoin carries serious regulatory risk. Finally, since the Bitcoin to dollar exchange rate has been so volatile, the investment also faces severe market risk. In the last several decades, the generalized autoregressive conditional heteroscedasticity $(\mathrm{GARCH})$ model has emerged as a standard tool for quantitative risk management. In this paper, we follow the trend and investigate the GARCH model in risk management of Bitcoin exchange rate returns. Our interests are on one of the special case of the generalized hyperbolic distribution, the normal reciprocal inverse Gaussian (NRIG) distribution, and we compare its empirical performance with the Student's $t$ distribution. Thus, our research is essentially similar as the work in Guo (2017a), but we focus on a very new asset class, Bitcoin, which has been rarely studied in the financial econometric literature. 


\section{Literature Review}

GARCH models with heavy-tailed distribution have been very frequently investigated in the literature, which covers a variety of asset classes. For instance, Bollerslev (1987) firstly developed a GARCH model with the Student's $t$ distribution and showed the model could explain stylized facts and dynamics of a variety of foreign exchange rates and stock price indices returns. Also investigating the stock market but focusing on individual securities, Tavares, Curto, and Tavares (2008) introduce the heavy tails and asymmetric effect on stocks returns volatility into the GARCH framework, and showed that the heavy-tailed distributions clearly outperform the normal distribution in fitting a series of the US stock returns. Guo (2017a) introduced several different types of heavy-tailed distribution into the GARCH models to compare their empirical performance in fitting a variety of individual stocks and stock indexes returns. Huang, Lee, and Liu (2008) and Guo (2017b) introduce the GARCH models with heavy-tailed distribution into the energy commodity market. Hung, Lee and Liu showed the Value at Risk estimates generated by the GARCH models have good accuracy at both low and high confidence levels. Guo showed that the GARCH models with heavy-tailed distributions could provide satisfactory margin calculations.

In this paper, we are interested in the Bitcoin exchange market and introduce the GARCH model with heavy-tailed distributions into this new special asset class. Although the literature on Bitcoin is limited overall, there are still several related studies. Dyhrberg (2016a) investigated the hedging capabilities of Bitcoin by applying the asymmetric GARCH methodology and showed that Bitcoin can clearly be used as a hedge against stocks in the Financial Times Stock Exchange Index and against the American dollar in the shortterm. Dyhrberg (2016b) explored the financial asset capabilities of Bitcoin using GARCH models and showed several similarities to gold and the dollar indicating hedging capabilities and advantages as a medium of exchange. Katsiampa (2017) explored the optimal conditional heteroscedasticity model with regards to goodness-of-fit to Bitcoin price data, and found that the best model is the AR-CGARCH model, indicating significance of including both a short-run and a long-run component of the conditional variance. Urquhart (2017) examined the volatility of Bitcoin as well as shedding light on the forecasting ability of GARCH models and heterogeneous auto-regressive (HAR) models in the Bitcoin market. Urquhart found no evidence of the leverage effect in Bitcoin and that the HAR models are superior in modeling Bitcoin volatility to traditional GARCH models.

None of the above literature has discussed the choice of heavy-tailed distributions within the GARCH framework in fitting the Bitcoin exchange rate returns. In this paper, we utilize the model framework in Guo (2017a) and compare the two types of heavy-tailed distributions, the Student's $t$ distribution and the NRIG distribution. The remainder of the paper is organized as follows. In Section 2, we discuss GARCH models and the heavy-tailed distributions. Section 3 summarizes the data. The estimation results are in Section 4. Finally, we conclude in Section 5.

\section{The Models}

A simple $\operatorname{GARCH}(1,1)$ process given by:

$$
\begin{aligned}
\varepsilon_{t} & =\mu+\sigma_{t} e_{t} \\
\sigma_{t}^{2} & =\alpha_{0}+\alpha_{1} \varepsilon_{t-1}^{2}+\beta_{1} \sigma_{t-1}^{2}
\end{aligned}
$$

where the three positive numbers $\alpha_{0}, \alpha_{1}$ and $\beta_{1}$ are the parameters of the process and $\alpha_{1}+\beta_{1}<1$. The assumption of a constant mean return $\mu$ is purely for simplification and reflects that the focus of the paper is on dynamics of return volatility instead of dynamics of returns. The variable $e_{t}$ is identically and independently distributed (i.i.d.). Firstly, we consider a benchmark case that $e_{t}$ follows a normal distribution:

$$
f\left(e_{t} \mid \psi_{t-1}\right)=\frac{1}{\sqrt{2 \pi \sigma_{t}^{2}}} \exp \left(-\frac{e_{t}^{2}}{2 \sigma_{t}^{2}}\right), v>4
$$

Then, we investigate two types of heavy-tailed distributions: the Student's $t$ and the normal reciprocal inverse Gaussian (NRIG) distributions. The density function of the standard Student's $t$ distribution with $v$ degrees of freedom is given by:

$$
f\left(e_{t} \mid \psi_{t-1}\right)=\frac{\Gamma\left(\frac{v+1}{2}\right)}{\Gamma\left(\frac{v}{2}\right)[(v-2) \pi]^{1 / 2}}\left(1+\frac{e_{t}^{2}}{(v-2)}\right)^{-\frac{v+1}{2}}, v>4 .
$$

where $\psi_{t-1}$ denotes the $\sigma$-field generated by all the available information up through time $t-1$.

The NRIG is a special class of the widely-used generalized hyperbolic distribution. The generalized hyperbolic distribution is specified as in Prause (1999): 


$$
f\left(e_{t} \mid \lambda, \mu, \alpha, \beta, \delta\right)=\frac{\left(\sqrt{\alpha^{2}-\beta^{2}} / \delta\right)^{\lambda} K_{\lambda-1 / 2}\left(\alpha \sqrt{\delta^{2}+\left(e_{t}-\mu\right)^{2}}\right)}{\sqrt{2 \pi}\left(\sqrt{\delta^{2}+\left(e_{t}-\mu\right)^{2}} / \alpha\right)^{1 / 2-\lambda} K_{\lambda}\left(\delta \sqrt{\alpha^{2}-\beta^{2}}\right)} \exp \left(\beta\left(e_{t}-\mu\right)\right),
$$

where $K_{\lambda}(\cdot)$ is the modified Bessel function of the third kind and index $\lambda \in \square$ and: $\delta>0,0 \leq|\beta|<\alpha$.

When $\lambda=\frac{1}{2}$, we have the normalized NRIG distribution as:

$$
f\left(\varepsilon_{t} \mid \psi_{t-1}\right)=\frac{\alpha K_{0}\left(\sqrt{\left(\alpha^{2}-1\right)^{2}+\frac{\alpha^{2} \varepsilon_{t}^{2}}{\sigma_{t}^{2}}}\right)}{\pi \sigma_{t}} \exp \left(\alpha^{2}-1\right) \text {. }
$$

\section{Data and Summary Statistics}

We investigate empirical performance of GARCH models with heavy-tailed distribution by using the daily Bitcoin exchange rate returns series. The daily Bitcoin exchange rates data are collected from Coindesk (www.coindesk.com) for the period from July 19, 2010 to July 23, 2017, covering all the available data in Coindesk. There are in total 2562 observations. Figure illustrates that the exchange rate of Bitcoin to USD is currently above $\$ 2700$, starting from almost nothing in 2010.

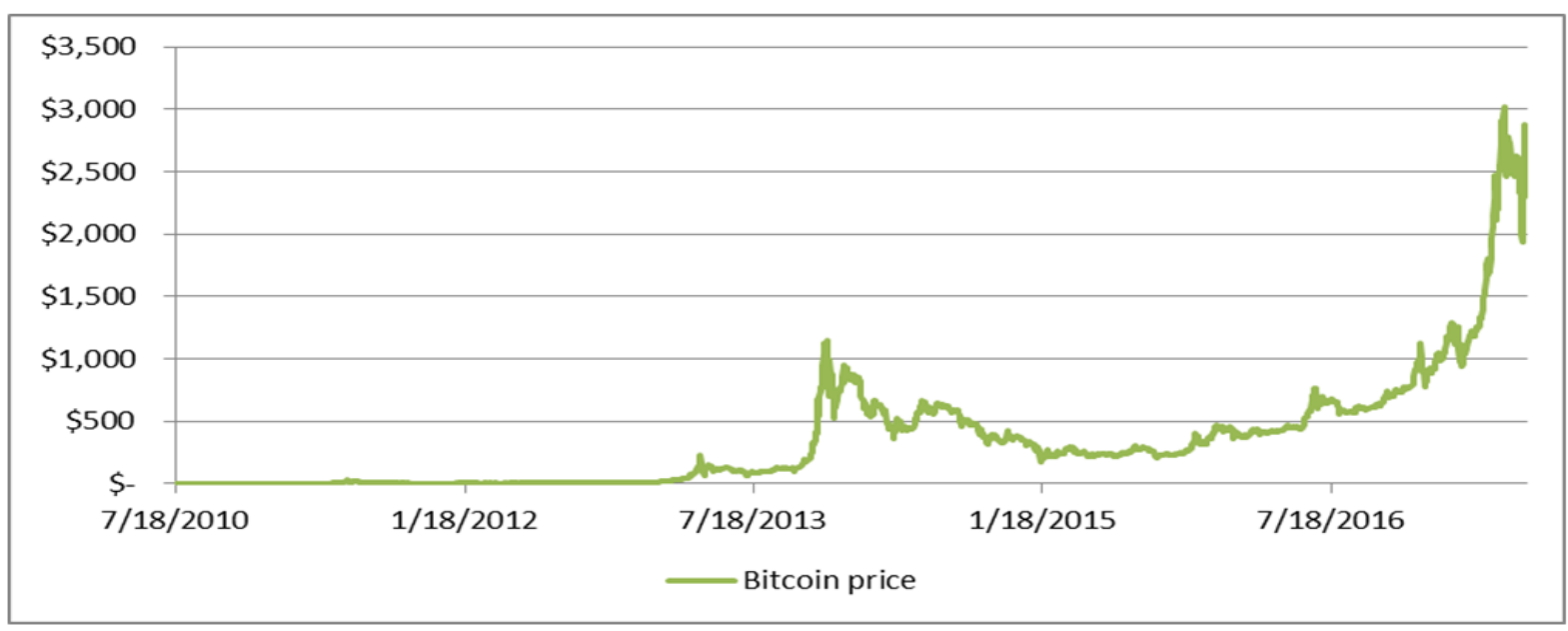

Figure-1. Daily Bitcoin exchange rate prices.

Figure 2 illustrates the dynamics of daily Bitcoin exchange rate returns. The daily exchange rate returns are very volatile and a lot of spikes are observed. The figure also exhibits significant volatility clustering.

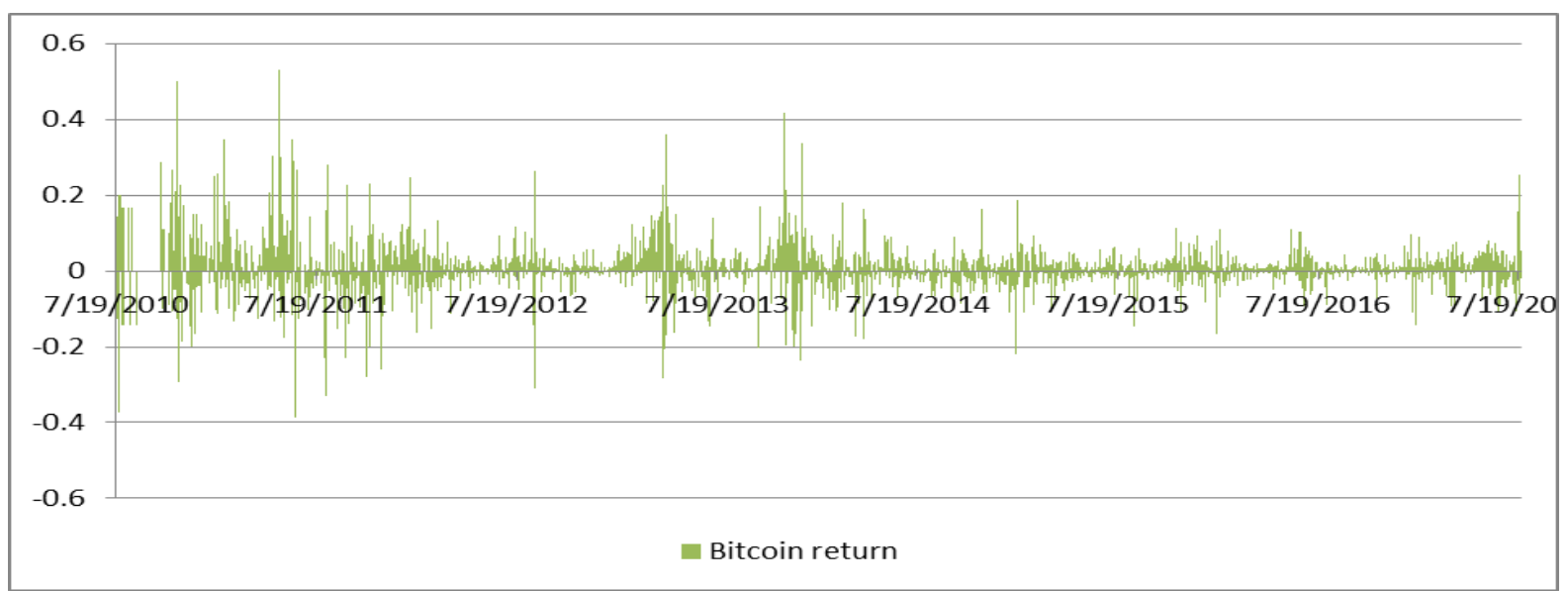

Figure-2. Daily Bitcoin exchange rate returns.

Summary statistics of the data are reported in Table 1. The data present the standard set of well-known stylized facts of asset prices series: non-normality, limited evidence of short-term predictability and strong evidence of predictability in volatility. All series are presented in daily percentage growth rates/returns. The 
Bera-Jarque test conclusively rejects normality of raw returns in all series, which confirms our assumption that the model selected should account for the heavy-tail phenomenon. The smallest test statistic is much higher than the $5 \%$ critical value of 5.99. The market index is negatively skewed and has fat tails. The asymptotic SE of the skewness statistic under the null of normality is $\sqrt{6 / T}$, and the SE of the kurtosis statistic is $\sqrt{24 / T}$, where $T$ is the number of observations. Almost all series exhibit statistically significant leptokurtosis, suggesting that accounting for heavy-tailedness is more pressing than skewness in modelling asset prices dynamics.

Table-1. Summary statistics. BJ is the Bera-Jarque statistic and is distributed as chi-squared with 2 degrees of freedom, $\mathrm{Q}(5)$ is the LjungBox Portmanteau statistic, $\mathrm{Q}^{\mathrm{ARCH}}(5)$ is the Ljung-Box Portmanteau statistic adjusted for ARCH effects following Diebold (1986) and $\mathrm{Q}^{2}(5)$ is the Ljung-Box test for serial correlation in the squared residuals. The three $\mathrm{Q}$ statistics are calculated with 5 lags and are distributed as chi-squared with 5 degrees of freedom.

\begin{tabular}{l|l|l|l|l|l|l|l|l|l}
\hline Series & Obs. & Mean & Std. & Skewness & Kurtosis & BJ & $Q_{(5)}$ & $Q^{\text {ARCH }}(\mathbf{5})$ & $\boldsymbol{Q}^{2}(\mathbf{5})$ \\
\hline $\begin{array}{l}\text { Daily Bitcoin } \\
\text { returns }\end{array}$ & 2562 & $0.58 \%$ & $5.95 \%$ & $0.86^{*}$ & $12.73^{* *}$ & $101.2^{* *}$ & $9.39^{*}$ & 4.37 & $37.41^{* *}$ \\
\hline
\end{tabular}

We use the Ljung-Box portmanteau, or $\mathrm{Q}$, statistic with five lags to test for serial correlation in the data, and adjust the $\mathrm{Q}$ statistic for ARCH models following Diebold (1986). The results that no serial correlation is found for almost all the series confirm our assumption of a constant mean return. The evidence of linear dependence in the squared demeaned returns, which is an indication of volatility clustering effects, is significant.

\section{Estimation Results}

To simplify the analysis, only the GARCH $(1,1)$ model with the Student's $t$ and the NRIG distributions is investigated. The model is estimated by maximizing the following log-likelihood function of equation:

$$
\hat{\theta}=\arg \max _{\theta} \sum_{t=1}^{T} \log \left(f\left(\varepsilon_{t} \mid \varepsilon_{1}, \cdots, \varepsilon_{t-1}\right)\right) \text {. }
$$

Table 2 reports estimation results of the $\operatorname{GARCH}(1,1)$ model with the two types of heavy-tailed distribution for all the daily Bitcoin exchange rate return series. All the parameters are significantly different from zero. There results show the Student's $t$ distribution has better in-sample performance. The Akaike information criterion (AIC) and the Bayesian information criterion (BIC) also indicate that the heavy-tailed distributions have better performance than the standard normal distribution and the Student's $t$ distribution has the best in-sample performance.

Table-2. Estimation of the GARCH model with heavy-tailed innovations

\begin{tabular}{l|l|l|l|l|l|l}
\hline & alpha 1 & beta 1 & 1/nu (1/alpha) & log-likelihood & AIC & BIC \\
\hline Normal & O.094** & 0.837** & & -5083 & 10170 & 10182 \\
\hline Student's t & O.101** & 0.802** & $0.423^{* *}$ & -4903 & 9811 & 9829 \\
\hline NRIG & $0.114^{* *}$ & $0.811^{* *}$ & $0.817^{*}$ & -4930 & 9867 & 9884 \\
\hline
\end{tabular}

* and ${ }^{*} *$ denote statistical significance at the $5 \%$ and $1 \%$ level respectively.

\section{Conclusion}

Since its first appearance, Bitcoin has gained more and more attention from either industry practitioners or academia. People are attracted by this new mysterious concept. Following the trend, we have compared empirical performance of a newly-developed heavy-tailed distribution, the normal reciprocal inverse Gaussian, with the most popular heavy-tailed distribution, the Student's $t$ distribution, under the GARCH framework in fitting the daily Bitcoin exchange rate returns. Our results indicate the heavy-tailed distribution has better performance in capture the daily Bitcoin exchange rate returns dynamics than the standard normal distribution. We further show the Student's $t$ distribution has the best in-sample performance. In this paper, we only consider the single Bitcoin market. It would be interesting to investigate the inter-connections between the Bitcoin market with some other markets using the GARCH framework as in Guo (2017c). We hope that will be another contribution to the literature on Bitcoin.

\section{References}

Bollerslev, T. (1987). A conditional heteroskedastic time series model for security prices and rates of return data. Review of Economics and Statistics, 69, 542-547.

Diebold, F. (1986). Testing for serial correlation in the presence of ARCH. Proceedings of the Business and Economic Statistics Section of the American Statistical Association, 3, 323-328.

Dyhrberg, A. (2016a). Hedging capabilities of Bitcoin. Is it the virtual gold? Finance Research Letters, 16, 139-144.

Dyhrberg, A. (2016b). Bitcoin, gold and the dollar - A GARCH volatility analysis. Finance Research Letters, 16, 85-92. 
Guo, Z. (2017a). Empirical performance of GARCH models with heavy-tailed innovations, Working Paper.

Guo, Z. (2017b). Models with short-term variations and long-term dynamics in risk management of commodity derivatives, Working Paper.

Guo, Z. (2017c). How information is transmitted across the nations? An empirical investigation of the US and Chinese commodity markets. Global Journal of Management and Business Research, 17, 1-11.

Huang, J., Lee, M., \& Liu, H. (2008). Estimation of value-at-risk for energy commodities via fat-tailed GARCH models. Energy Economics, 8, 1173-1191.

Katsiampa, P. (2017). Volatility estimation for Bitcoin: A comparison of GARCH models. Economics Letters, 158, 3-6.

Prause, K. (1999). The generalized hyperbolic model: Estimation, financial derivatives, and risk measures. Ph.D. Dissertation.

Tavares, A., Curto, J., \& Tavares, G. (2008). Modelling heavy tails and asymmetry using ARCH-type models with stable Paretian distributions. Nonlinear Dynamics, 51(1), 231-243.

Urquhart, A. (2017). The volatility of Bitcoin, Working Paper. 